\title{
Do three-dimensional modeling and printing technologies have an impact on the surgical success of percutaneous transsacral screw fixation?
}

\author{
Serhat Durusoy, $\mathrm{MD}^{1}{ }^{10}$, Volkan Akdoğan, $\mathrm{MD}^{2}{ }^{(1)}$, Ahmet Emre Paksoy, $\mathrm{MD}^{1}$ \\ ${ }^{1}$ Department of Orthopedics and Traumatology, Yozgat Bozok University Faculty of Medicine, Yozgat, Turkey \\ ${ }^{2}$ Department of Electrical and Electronics, Yozgat Bozok University Faculty of Engineering, Yozgat, Turkey
}

Caused by high-energy traumas, pelvic injuries are life-threatening complex injuries posing several unfavorable effects on the body. Pelvic fractures account for approximately $3-4.3 \%$ of all skeletal fractures. ${ }^{[1,2]}$ Mortality rates due to pelvic injuries vary from 10 to $16 \% \cdot{ }^{[1,3]}$ Victims of these injuries display an increased tendency to develop complications due to the nature of the injury and modes of treatment. ${ }^{[4-8]}$ Complications are critical for patient survival and sequelae development.

Percutaneous iliosacral and transsacral-transiliac screw fixation are the commonly preferred surgical methods for the treatment of posterior pelvic injuries. The advantages of this technique include minimal tissue damage due to the surgical procedure, improved stability of the fixation, short surgery time, and reduced risk for wound-related problems in the postoperative period. ${ }^{[9,10]}$ However, patients may be prone to develop complications due to some factors, including variability in pelvic anatomy, the narrowness of the bone corridor to be screwed, and inadequacies in imaging and

Received: December 27, 2019

Accepted: February 12, 2020

Published online: June 18, 2020

Correspondence: Serhat Durusoy, MD. Yozgat Bozok Üniversitesi Tıp Fakültesi Eğitim ve Araştırma Hastanesi Ortopedi ve

Travmatoloji Kliniği, 66900 Yozgat, Türkiye.

E-mail: serquat@gmail.com

Doi: $10.5606 /$ ehc. 2020.73115

Citation: Durusoy S, Akdoğan V, Paksoy AE. Do three-dimensional modeling and printing technologies have an impact on the surgical success of percutaneous transsacral screw fixation? Jt Dis Relat Surg 2020;31(2):273-280.

\section{ABSTRACT}

Objectives: This study aims to determine the role of computed tomography (CT)-derived templates, produced by threedimensional (3D) modeling, image processing and printing technology, in percutaneous transsacral screw fixation and evaluate the effects of their use on surgical success.

Materials and methods: This prospective study conducted between June 2018 and December 2019 utilized 15 composite pelvis models for transsacral-transiliac screw fixation. For the procedure, modeled templates were utilized for wiring on the left side of the pelvis models, while the conventional method was performed on the right side of the pelvis models. In the computed tomography images acquired after wiring, appropriate wire position was evaluated.

Results: The placed wires held the S1 body appropriately in all of the procedures with or without template use. With the template use, the wires were placed appropriately in the surgical bone corridor suitable for the transsacral-transiliac screw fixation in all of the models. However, with the conventional methods, the wires were not placed in the safe surgical bone corridor in four models. The wire deviation angle in the axial plane was significantly lower in the template group $(\mathrm{p}=0.001)$, whereas it was not different between the template group and the conventional method group in the coronal plane $(\mathrm{p}=0.054)$. The amount of deviation from the ideal wire entry site was significantly reduced in the template group compared to the conventional method group $(p=0.001)$.

Conclusion: With the use of 3D modeling and printing technology, CT-derived templates can be produced and utilized for transsacral screw fixation procedures and their use increases surgical success by reducing the surgical margin of error.

Keywords: Image processing, patient-specific guide, threedimensional modeling, three-dimensional printing technology, transsacral-transiliac screw fixation.

surgical procedures. ${ }^{[1]]}$ In the literature, the current technique has been modified by combining with different imaging techniques in order to decrease the complication rates and increase surgical success. 
Computed tomography (CT) and computerized navigation systems are the most common combinations introduced for this purpose. It has been reported that the success of surgery is improved when these imaging methods are used in iliosacral and transsacral-transiliac screw fixation. However, applicability, cost, and need for additional experience are the discussed aspects of these methods.

Several industrial sectors (engineering, biomedical, medicine, industrial, space technologies, etc.) have already adopted three-dimensional (3D) modeling and printing technology into their daily practices. In medicine, this technology is used particularly in maxillofacial and spinal surgery, while its use is not common in orthopedics. In this study, we aimed to determine the role of CT-derived templates, produced by 3D modeling, image processing and printing technology, in percutaneous transsacral screw fixation and evaluate the effects of their use on surgical success.

\section{MATERIALS AND METHODS}

A total of 15 composite pelvis models were used for transsacral-transiliac fixation in our prospective study conducted between June 2018 and December 2019.
Guiding Kirschner (K)-wires were used on both sides of the composite pelvis models for transsacraltransiliac screwing. Three-dimensional modeling templates were used for the fixation on the left side. The conventional method was used on the right side.

The models were created by an engineer specialized in image processing. A modeling program, a free and open-source 3D creation suite, was utilized for modeling. During the surgical procedures, the models were placed in a radiolucent foam mold and covered with a surgical drape.

The templates created for the study were modeled in two parts (Figure 1a, b). The first part of each template was fixed and it was for use in all pelvic systems. The second part was modular and modeled for each pelvis specifically.

The fixed part of the template had the holes and sleeves through which the guiding K-wires passed so that the template would be prevented from rotating and would be placed at an appropriate distance to the bone surface. The fixed part of the template had one long and one short sleeve. The long sleeve was used for keeping the template at an appropriate distance from the bone surface (Figure 2b). The

(a)
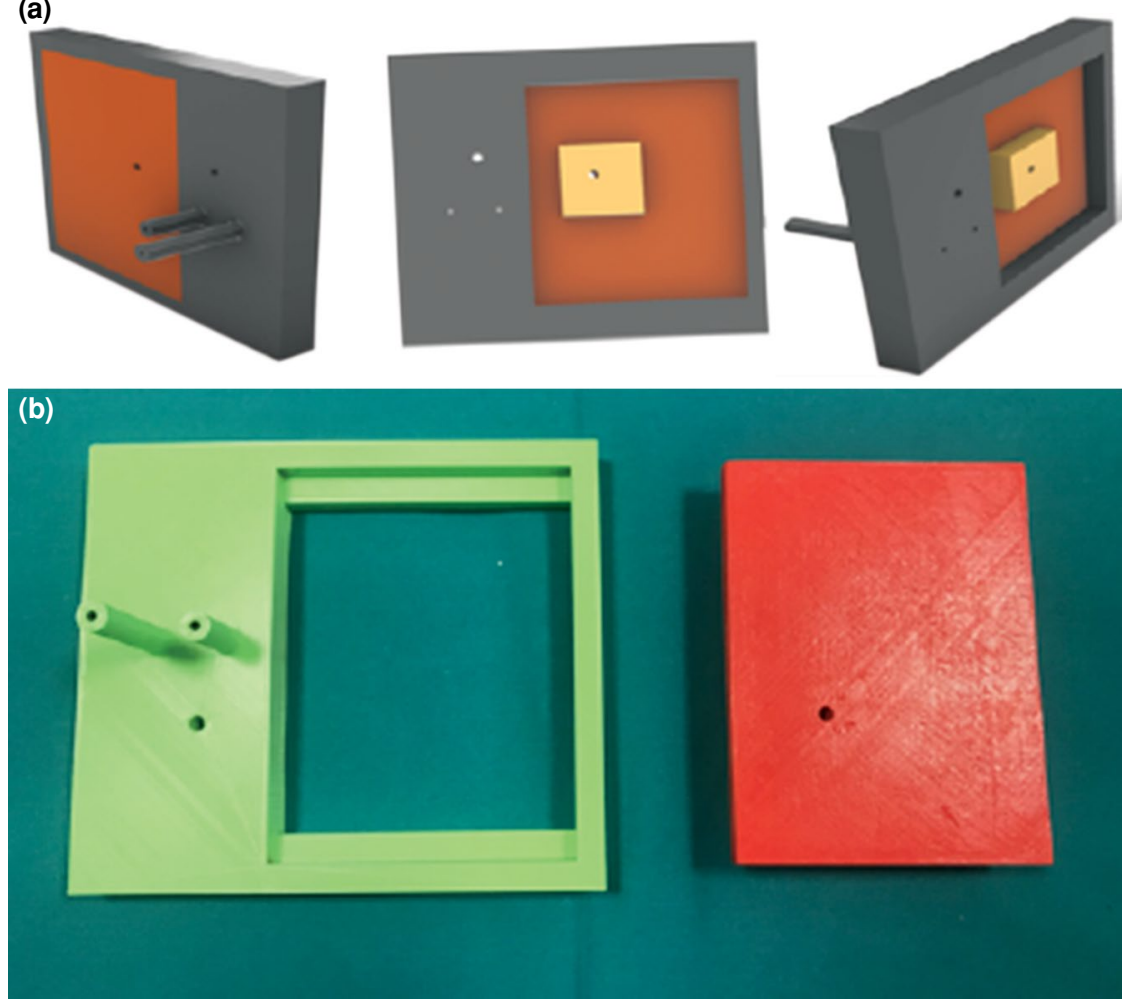

FIGURE 1. (a) Three-dimensional schematic views of templates. (b) Real appearance of modeled templates. 
short sleeve was designed for the second K-wire to pass through to prevent the rotation of the template. This piece of the template had no contact points with the bone surface. Also, a hole, through which a $2.5 \mathrm{~mm}$-diameter sleeve can pass, was designed on the fixed part of the template to ensure further stability of the system. The use of this hole was at the surgeon's discretion. A $145 \times 110 \times 20 \mathrm{~mm}$ groove was designed on the fixed part for the modular part to fit into. In this study, three K-wires were inserted through the sleeves and hole in the fixed part of the template to fix the supraacetabular region in all pelvis models. These K-wires ensured the stability of the 3D spatial position of the template relative to the pelvis. Then, CT images of the pelvis models were acquired.

The modular part was fabricated after acquiring the 3D models based on the CT images of each pelvis. Then, the obtained 3D images were uploaded to the $3 \mathrm{D}$ modeling program. In this program, the previously fabricated fixed part of the template was brought to the appropriate position by using the guiding $\mathrm{K}$-wires in the supraacetabular region as reference (Figure 2). The modular part was then designed to be placed on groove on the fixed part. The ideal orientation and entry point for the guiding $\mathrm{K}$-wire were determined on the axial and coronal views of the CT images (Figure 3). Using these ideal orientation and entry point, the hole designed for the guiding K-wire to pass through was placed on the designed module. The orientation of this hole was specifically adjusted for each pelvis on the spatial axes by using the 3D modeling and image processing program. Modular parts, specifically designed for each pelvis model, were printed using a 3D printer.

(a)
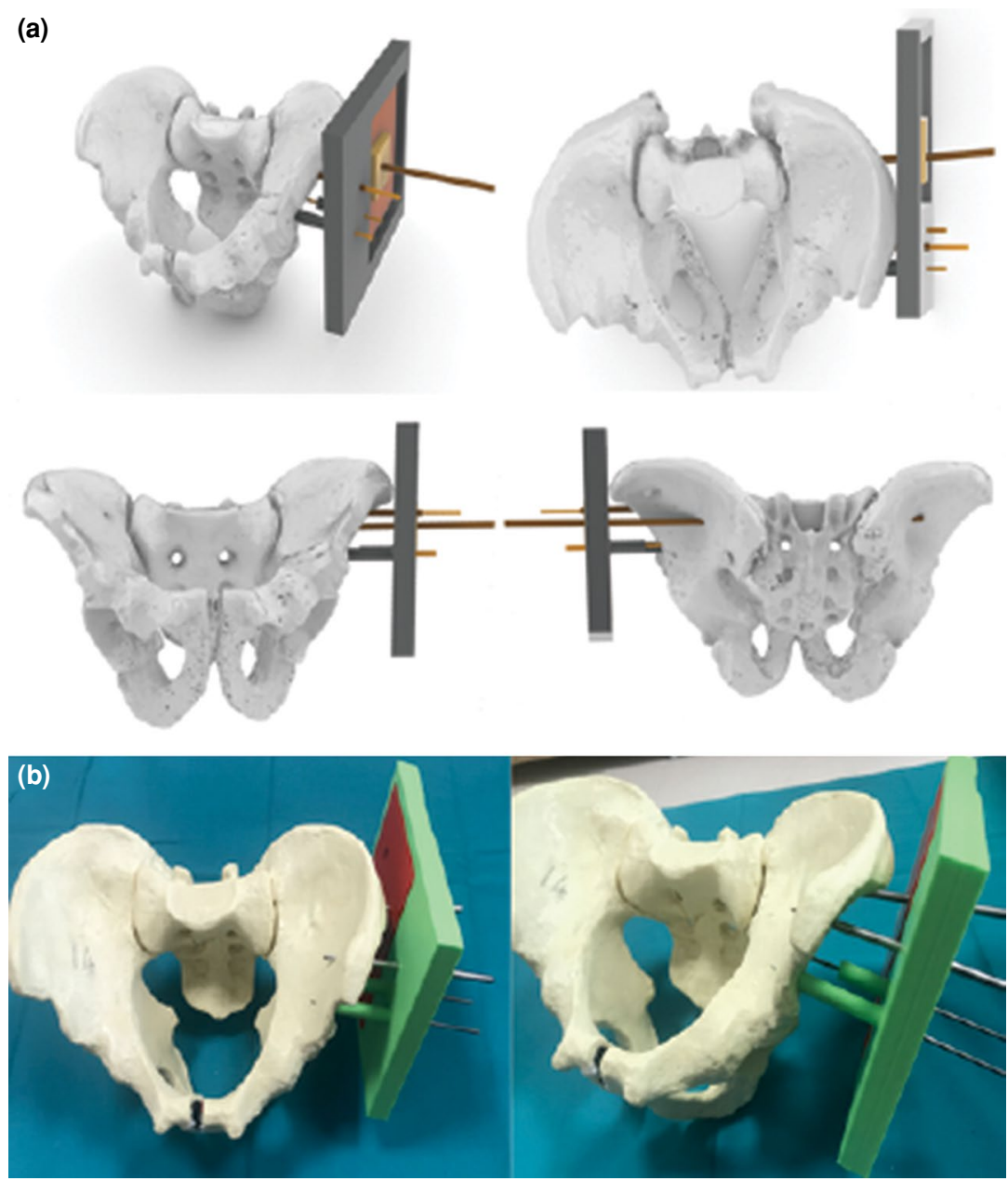

FIGURE 2. (a) Three-dimensional schematic view of application of modular part to fixed part and pelvis model. (b) Real view of application of modular part to fixed part and pelvis model. 

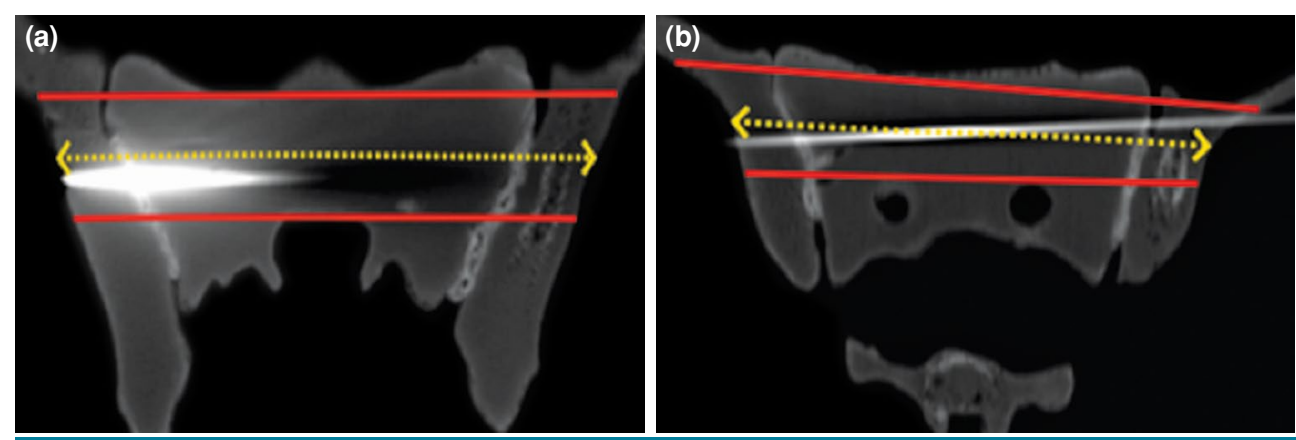

FIGURE 3. Determination of proper screw placement and insertion on axial and coronal images of computed tomography.
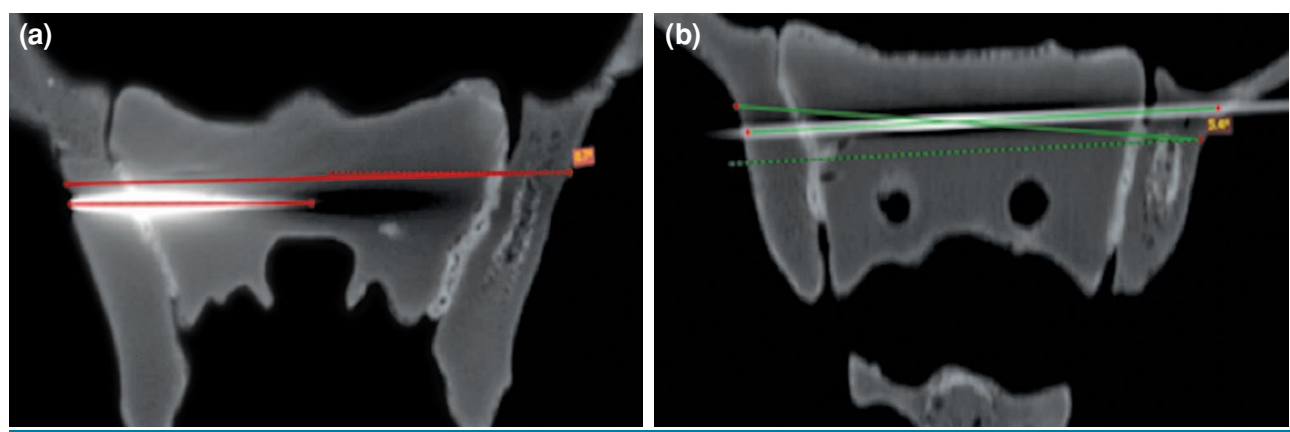

FIGURE 4. Determination of deviation angle from appropriate Kirschner-wire orientation on axial plane (a) and coronal plane (b) computed tomography images.

The modular parts used for the left side wiring were mounted on the fixed part of template. The template was then placed on the guiding K-wires, which were previously sent supraacetabularly, and wiring was made over the modular part with scopy. On the right side, the guiding K-wires were passed through under scopy using the conventional method.

The success of the study was evaluated by using the CT images acquired after the procedure. The following were evaluated:

1. Whether they hold the S1 vertebrae body within the confines of the safe bone corridor,

2. Whether they reach the transsacral contralateral iliac bone within the limits of the safe bone corridor,

3. The deviation angle from the appropriate guiding K-wire orientation, determined previously on the axial plane CT images (Figure 4a),

4. The deviation angle from the appropriate guiding K-wire orientation, pre-determined on the coronal plane CT images (Figure $4 b$ ),
5. The distance ("a" distance) between the entry point and the pre-determined ideal entry point for the guiding K-wire (Figure 5).

Also, the cost of each template was calculated.

\section{Statistical analysis}

JASP version 0.12.2 software (JASP Team, 2020, University of Amsterdam) was used for analyses. Firstly, descriptive statistics were performed. The Mann-Whitney U test was used as a non-parametric test for determining the differences between the groups. A $p$ value of $<0.05$ was accepted to indicate a statistically significant difference.

\section{RESULTS}

The guiding K-wires were successfully placed in S1 vertebral bodies for both groups in all sacroiliac screw fixation models. Guiding K-wire retention status of the contralateral iliac bone (transsacraltransiliac screw fixation) was $15 / 15(100 \%)$ in the template models and $11 / 15(73.3 \%)$ in the conventional method group. In the latter group, in four applications (26.7\%), the guiding K-wires passed beyond the margins of the safe corridor after passing through the S1 body (Table I). 


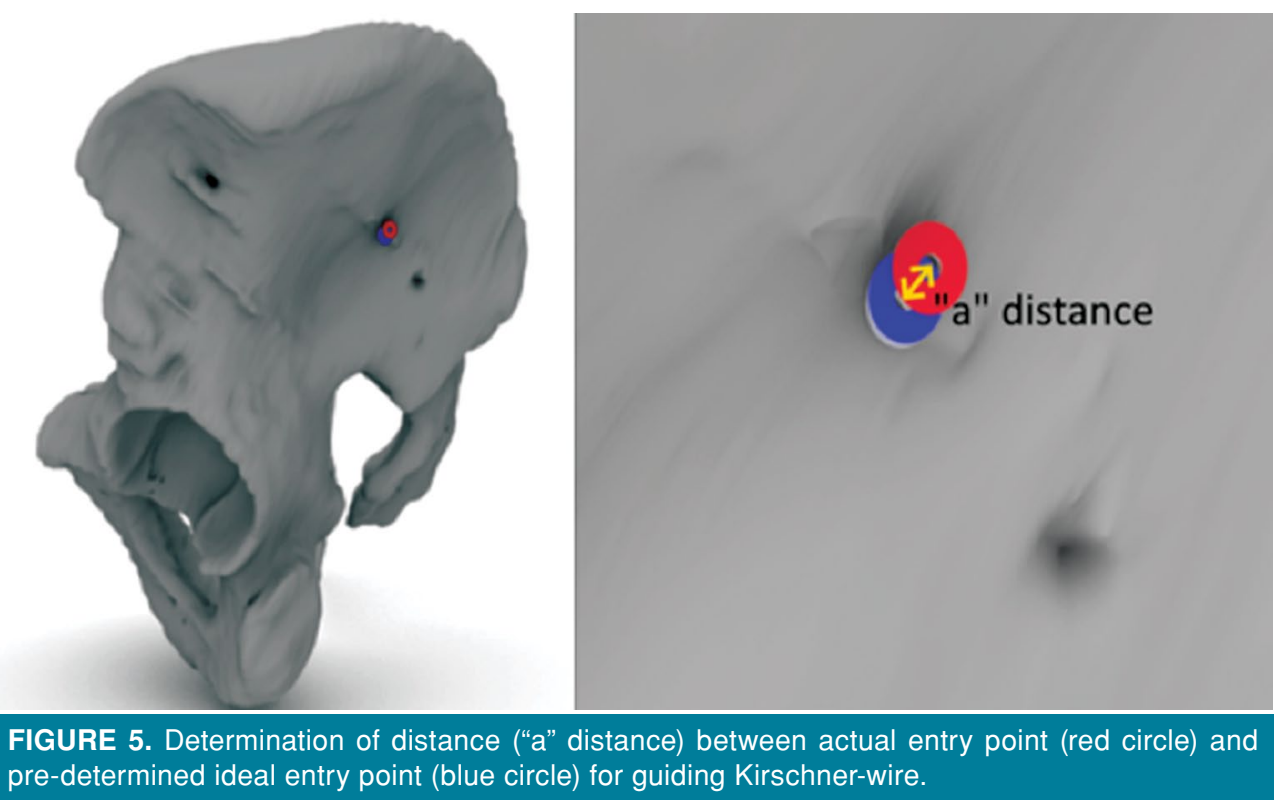

The axial and coronal CT images acquired after the procedure were examined to determine the deviation angle. In the axial $\mathrm{CT}$ images, the mean deviation angle was $2.68^{\circ}\left(0.4^{\circ}-6.3^{\circ}\right)$ in the template group and $6.38^{\circ}\left(1^{\circ}-17.6^{\circ}\right)$ in the conventional method group. The difference in the axial deviation angle between the two groups was statistically significant (Table I) $(\mathrm{p}=0.001)$. In the coronal images, the mean deviation angle was $2.17^{\circ}\left(0.3^{\circ}-4.4^{\circ}\right)$ in the template group and $4.32^{\circ}\left(1^{\circ}-18.4^{\circ}\right)$ in the conventional method group. The difference in the coronal deviation angle between the groups was not statistically significant (Table I) $(\mathrm{p}=0.054)$.

The mean distances between the actual and predetermined entry points of the guiding K-wires were $1.43 \mathrm{~mm}$ (range, $0-4 \mathrm{~mm}$ ) in the template group and $5.97 \mathrm{~mm}$ (range, 0.06-11 $\mathrm{mm}$ ) in the conventional method group. The difference between the two groups was statistically significant (Table I) $(\mathrm{p}=0.001)$.

The cost required for the fabrication of one 3D template was found to be 71.33 Turkish liras (12.4 USD).

\section{DISCUSSION}

Iliosacral and transsacral-transiliac screw fixations are commonly used for the treatment of posterior pelvic injuries because they are minimally invasive. ${ }^{[12]}$ In the iliosacral screw fixation, the iliac wing is the entry point and the sacral vertebra body is the target. In transacral-transiliac screw fixation, the entry point is the iliac wing, while the target point is the opposite iliac wing. In the latter technique, the fixation screw bypasses the body of the sacral vertebra. In transsacral-transiliac screw fixation, both the safe

\section{TABLE I}

Statistical analyses of evaluation criteria

\begin{tabular}{|c|c|c|c|c|c|c|c|c|c|}
\hline & \multicolumn{4}{|c|}{ Wiring with template } & \multicolumn{4}{|c|}{ Wiring with conventional method } & \multirow[b]{2}{*}{$p^{*}$} \\
\hline & $\mathrm{n}$ & $\%$ & Mean & Min-Max & $\mathrm{n}$ & $\%$ & Mean & Min-Max & \\
\hline Rate of wire placement in S1 vertebra body & $15 / 15$ & 100 & & & $15 / 15$ & 100 & & & \\
\hline Applicability for transsacral screwing & $15 / 15$ & 100 & & & $11 / 15$ & 73.3 & & & \\
\hline Deflection angle in axial plane & & & $2.68^{\circ}$ & $0.4^{\circ}-6.3^{\circ}$ & & & $6.38^{\circ}$ & $1^{\circ}-17.6^{\circ}$ & 0.001 \\
\hline Deflection angle in coronal plane & & & $2.17^{\circ}$ & $0.3^{\circ}-4.4^{\circ}$ & & & $4.32^{\circ}$ & $1^{\circ}-18.4^{\circ}$ & 0.054 \\
\hline $\begin{array}{l}\text { Amount of deviation from the } \\
\text { ideal entry point }(\mathrm{mm})\end{array}$ & & & 1.43 & $0-4$ & & & 5.97 & $0.06-11$ & 0.001 \\
\hline
\end{tabular}


surgical bone corridor and the angular error margin are narrower compared to those used in the iliosacral screw fixation. However, in the literature, it has been demonstrated that the stability is higher with the transsacral-transiliac screw fixation compared to the iliosacral screw fixation technique. ${ }^{[13]}$ Both of these surgical techniques are frequently used in practice today conventionally with the guidance of fluoroscopy. Complications associated with the application of these techniques occur due to variations in the pelvic anatomy, narrow surgical fields, inadequacies of the imaging methods, and inadequate surgical experience. ${ }^{[14-18]}$ There is an increasing number of studies suggesting combination with other imaging techniques to reduce complication rates and achieve better surgical results. The most commonly reported combination is the use of CT with two-dimensional (2D)/3D image-based navigation systems.

Pieske et al. ${ }^{[19]}$ demonstrated improved safety of the surgery when $\mathrm{CT}$ was used in iliosacral screw fixation. Zwingmann et al. ${ }^{[20]}$ confirmed the findings of Pieske et al. ${ }^{[19]}$ Zwingmann et al. ${ }^{[20]}$ reported a shorter time of irradiation and lower doses in the CT navigation group compared to the control group. Also, they reported that the screw placement results were more favorable in the navigated group. However, the duration of the procedure was not different between the groups. In another study, Zwingmann et al., ${ }^{[21]}$ compared the rates of inaccurate screw positioning among the conventional method, 2D/3D navigation method, and the $\mathrm{CT}$ navigation method, reporting that the screw was incorrectly positioned at rates of $2.6 \%, 1.3 \%$, and $0.1 \%$ in those groups, respectively. Takao et al. ${ }^{[22]}$ demonstrated improved success rates with a CT-3D-fluoroscopy combination in screw fixation procedures. This combined method certainly improves the success of fixation; however, the critical concerns associated with this combination needs to be worked through, including the maintenance of the sterile environment during the procedure, convenient accessibility to CT facilities, the narrowness of the inside area of $\mathrm{CT}$, and irradiation exposure.

Navigation-assisted imaging techniques provide alternative ways to the aforementioned methods in minimizing the complications and margin of error. In the literature, the use of these navigation systems has been associated with improved success rates and reduced duration of insertion and irradiation exposure time in transiliac-transsacral screw fixation. ${ }^{[17,23]}$ Thakkar et al. ${ }^{[16]}$ reported better success rates with the use of the $3 \mathrm{D}$ navigation system in screw fixation compared to the conventional method. Contrary to the findings reported by those studies, Collinge et al. ${ }^{[24]}$ could not show differences in the surgical success rates when the standard multiplanar fluoroscopy was compared to the use of the fluoroscopy-based navigation system. However, they reported that the duration of the screw fixation procedure and the level of the exposed irradiation were reduced with the use of the navigation system. Zwingman et al. ${ }^{[25]}$ reported the most interesting study, criticizing the navigation systems. They compared the 2D/3D navigation and conventional methods for the intra- and postoperative complication rates, reporting no statistically significant differences between the two groups; however, the intraoperative complication rate was less in the conventional group compared to the navigation group. These studies demonstrate that achieving success with the use of navigation systems remains to be under debate. Furthermore; the accessibility, applicability, and cost of these systems are critical and await further clarification. ${ }^{[17]}$

In our study, we evaluated the use of templates in transsacral-transiliac screw fixation. After the templates were designed for each pelvisindividually, they were fabricated with $3 \mathrm{D}$ printing machines for use. The number of studies investigating the use of templates produced by 3D modeling is limited in the literature. Chen et al. ${ }^{[26]}$ used specially modeled personal image-based templates in their study. They reported that the screw positioning was improved, the duration of surgery was short, and the time of exposure to irradiation was reduced in the template group. Yang et al. ${ }^{[27]}$ confirmed those findings by demonstrating favorable screw location and reduced time for the procedure and irradiation exposure in their study. Liu et al. ${ }^{[28]}$ investigated the use of $3 \mathrm{D}$ printed templates in sacroiliac screw fixation, finding out that the time required for the surgeon to gain experience was reduced and the feasibility of the surgery became favorable. Zhou et al. ${ }^{[29]}$ compared the use of 3D templates with the conventional method. They reported that compared to the conventional method, the duration of the procedure and the quantity of exposed irradiation were reduced and the reliability of the surgery was improved with the use of 3D printed templates.

Our study findings confirm the results reported by the previous studies discussed above. The guiding K-wires were found in the safe surgical bone corridor for the transsacral-transiliac screw fixation in all of the 3D-modeled templates. However, in the conventional group, the guiding K-wires 
deviated from the safe surgical bone corridor in four applications (26.7\%) and they were not found in an optimum position for the transsacral-transiliac screw fixation. Furthermore, in the template group, the deviation angle from the ideal guiding K-wire axis and distance of the deviation from the ideal guiding K-wire entry point on the axial plane were less compared to those found in the conventional method group. These findings support that the use of CT-derived 3D templates improves the success rate of the transsacral-transiliac screw fixation. Our study is different from the previous studies which used designed templates for mini open surgery. In our study, we specifically designed and used the 3D templates for the percutaneous surgical technique. When examined in terms of cost, the cost per patient was approximately 12.4 USD. This amount is quite reasonable considering surgical complications and the resulting costs.

The small sample size is the limitation of our study. Further large-scale studies elaborating the use of CT-derived model-based templates in transsacraltransiliac screw fixation are needed. We believe that the method will be implemented into the surgical practice based on the results to be reported by future studies. A critical aspect of our study is the required know-how to use the modeling programs to create the templates. In our study, an engineer, specialized in image processing, designed the model and fabricated the templates. We think that the development of simple software specifically designed for creating CT-derived models to fabricate templates for the transsacral-transiliac screw fixation procedure will allow implementing this method to common surgical practice.

In conclusion, our study has found out that the use of patient-specific CT-derived 3D model-based templates in transsacral-transiliac screw fixation improves the accuracy of screw placement and orientation, increases the surgical success rate, and decreases the potential to develop complications. The potential costs associated with this procedure are reasonable. In addition, we predict that the exposed radiation doses caused by scopy will be decreased significantly by using this method.

\section{Declaration of conflicting interests}

The authors declared no conflicts of interest with respect to the authorship and/or publication of this article.

\section{Funding}

This study was supported by the Scientific Research and Project Coordination Center of Yozgat Bozok University (project number: 6602c-TF/18-210)

\section{REFERENCES}

1. Hermans E, Biert J, Edwards MJR. Epidemiology of Pelvic Ring Fractures in a Level 1 Trauma Center in the Netherlands. Hip Pelvis 2017;29:253-61.

2. Swaid F, Peleg K, Alfici R, Olsha O, Givon A, Kessel B, et al. A comparison study of pelvic fractures and associated abdominal injuries between pediatric and adult blunt trauma patients. J Pediatr Surg 2017;52:386-9.

3. Grotz MR, Allami MK, Harwood P, Pape HC, Krettek C, Giannoudis PV. Open pelvic fractures: epidemiology, current concepts of management and outcome. Injury 2005;36:1-13.

4. van den Bosch EW, van Zwienen $C M$, van Vugt $A B$. Fluoroscopic positioning of sacroiliac screws in 88 patients. J Trauma 2002;53:44-8.

5. Routt ML Jr, Kregor PJ, Simonian PT, Mayo KA. Early results of percutaneous iliosacral screws placed with the patient in the supine position. J Orthop Trauma 1995;9:207-14.

6. Tonetti J, Cazal C, Eid A, Badulescu A, Martinez T, Vouaillat $\mathrm{H}$, et al. Neurological damage in pelvic injuries: a continuous prospective series of 50 pelvic injuries treated with an iliosacral lag screw. Rev Chir Orthop Reparatrice Appar Mot 2004;90:122-31.

7. Schweitzer D, Zylberberg A, Córdova M, Gonzalez J. Closed reduction and iliosacral percutaneous fixation of unstable pelvic ring fractures. Injury 2008;39:869-74.

8. Osterhoff G, Noser J, Sprengel K, Simmen HP, Werner CML. Rate of intraoperative problems during sacroiliac screw removal: expect the unexpected. BMC Surg 2019;19:39.

9. Lucas JF, Routt ML Jr, Eastman JG. A useful preoperative planning technique for transiliac-transsacral screws. J Orthop Trauma 2017;31:e25-e31.

10. Lindsay A, Tornetta P 3rd, Diwan A, Templeman D. Is closed reduction and percutaneous fixation of unstable posterior ring injuries as accurate as open reduction and internal fixation? J Orthop Trauma 2016;30:29-33.

11. Durusoy S, Paksoy AE, Korkmaz M, Solak AŞ, Dağlar B. Is pelvic mapping applicable in iliosacral screw fixation to determine screw entry point and screw trajectory? Eklem Hastalik Cerrahisi 2019;30:252-8.

12. Tidwell J, Cho R, Reid JS, Boateng H, Copeland C, Sirlin E. Percutaneous Sacroiliac Screw Technique. J Orthop Trauma 2016;30:S19-20.

13. Gardner MJ, Routt ML Jr. Transiliac-transsacral screws for posterior pelvic stabilization. J Orthop Trauma 2011;25:378-84.

14. Aprato A, Devivo S, Masse A. Ten tips to improve iliosacral screw placement. J Clin Exp Orthop 2018;4:59.

15. Routt ML Jr, Simonian PT, Mills WJ. Iliosacral screw fixation: early complications of the percutaneous technique. J Orthop Trauma 1997;11:584-9.

16. Thakkar SC, Thakkar RS, Sirisreetreerux N, Carrino JA, Shafiq B, Hasenboehler EA. 2D versus 3D fluoroscopybased navigation in posterior pelvic fixation: review of the literature on current technology. Int J Comput Assist Radiol Surg 2017;12:69-76.

17. Khan JM, Lara DL, Marquez-Lara A, Rosas S, Hasty E, Pilson HT. Intraoperative CT and Surgical Navigation for Iliosacral Screws: Technique for Patients With Sacral Dysmorphism. J Orthop Trauma 2018;32:S24-S5. 
18. Miller AN, Routt ML Jr. Variations in sacral morphology and implications for iliosacral screw fixation. J Am Acad Orthop Surg 2012;20:8-16.

19. Pieske O, Landersdorfer C, Trumm C, Greiner A, Wallmichrath J, Gottschalk O, et al. CT-guided sacroiliac percutaneous screw placement in unstable posterior pelvic ring injuries: accuracy of screw position, injury reduction and complications in 71 patients with 136 screws. Injury 2015;46:333-9.

20. Zwingmann J, Konrad G, Kotter E, Südkamp NP, Oberst M. Computer-navigated iliosacral screw insertion reduces malposition rate and radiation exposure. Clin Orthop Relat Res 2009;467:1833-8.

21. Zwingmann J, Hauschild O, Bode G, Südkamp NP, Schmal $\mathrm{H}$. Malposition and revision rates of different imaging modalities for percutaneous iliosacral screw fixation following pelvic fractures: a systematic review and metaanalysis. Arch Orthop Trauma Surg 2013;133:1257-65.

22. Takao M, Nishii T, Sakai T, Yoshikawa H, Sugano N. Iliosacral screw insertion using CT-3D-fluoroscopy matching navigation. Injury 2014;45:988-94.

23. Li B, He J, Zhu Z, Zhou D, Hao Z, Wang Y, et al. Comparison of 3D C-arm fluoroscopy and 3D image-guided navigation for minimally invasive pelvic surgery. Int J Comput Assist Radiol Surg 2015;10:1527-34.

24. Collinge C, Coons D, Tornetta P, Aschenbrenner J.
Standard multiplanar fluoroscopy versus a fluoroscopically based navigation system for the percutaneous insertion of iliosacral screws: a cadaver model. J Orthop Trauma 2005;19:254-8.

25. Zwingmann J, Südkamp NP, König B, Culemann U, Pohlemann T, Aghayev E, et al. Intra- and postoperative complications of navigated and conventional techniques in percutaneous iliosacral screw fixation after pelvic fractures: Results from the German Pelvic Trauma Registry. Injury 2013;44:1765-72.

26. Chen B, Zhang Y, Xiao S, Gu P, Lin X. Personalized imagebased templates for iliosacral screw insertions: a pilot study. Int J Med Robot 2012;8:476-82.

27. Yang F, Yao S, Chen KF, Zhu FZ, Xiong ZK, Ji YH, et al. A novel patient-specific three-dimensional-printed external template to guide iliosacral screw insertion: a retrospective study. BMC Musculoskelet Disord 2018;19:397.

28. Liu Y, Zhou W, Xia T, Liu J, Mi BB, Hu LCet al. Application of the guiding template designed by three-dimensional printing data for the insertion of sacroiliac screws: a new clinical technique. Curr Med Sci 2018;38:1090-5.

29. Zhou W, Xia T, Liu Y, Cao F, Liu M, Liu J, et al. Comparative study of sacroiliac screw placement guided by 3D-printed template technology and X-ray fluoroscopy. Arch Orthop Trauma Surg 2020;140:11-7. 